\title{
Multidisciplinary Approach for the Management of a Case of Bilateral Cleft Lip and Palate (Baru's Score 5) Using Modified Dentoalveolar Distractor
}

\author{
Swapnaja Gosavi ${ }^{1}$, Sunita Shrivastav² ${ }^{2}$ Ranjit Kamble ${ }^{3}$, Himija Karia ${ }^{4}$, Nitin Bhola ${ }^{5}$ \\ 1, 2, 3, 4, 5 Department of Orthodontics, Sharad Pawar Dental College, Sawangi Meghe, Maharashtra, India. \\ ${ }^{4}$ Department of Orthodontics, VSPM Dental College, Nagpur, Maharashtra, India.
}

\section{PRESENTATION OF CASE}

A 19-year-old female reported to the Department of Orthodontics of Sharad Pawar Dental College and Hospital Maharashtra, India, with chief complaints of poor aesthetics and forwardly placed teeth. Clinical evaluation showed repaired bilateral CLP (Cleft Lip and Palate). Surgical scars were seen on upper lips. Lower lip was everted and ahead of upper lip with positive lip step. Profile was concave with increased clinical FMA (Frankfort-Mandibular plane Angle). She had apparently symmetrical leptoprosopic face form and competent lips as shown in Figure 1.

Intra oral examination revealed that over retained 51,53, 63 were present, both maxillary lateral Incisor and left canine were missing clinically. Rest all other teeth were present in both arches except third molars. Oronasal fistula and macroglossia was observed as shown in Figure 2. Angle's class III molar relationship with constricted maxillary arch having ' $v$ ' shape of upper and lower arch. Arches with crowding was present. Cephalometric analysis revealed skeletal class III with vertical growth pattern due to short maxilla and large prognathic mandible with slightly retroclined incisors. Panoramic radiograph revealed impaction with 23.

Management of cleft cases require multidisciplinary approach and teamwork. Several aesthetic and functional problems are associated with cleft lip and palate. ${ }^{1}$ One of the challenges faced is the severity of skeletal defect and availability of healthy bone as well as tissue. Orthodontist play a role in treatment planning at various stages of cleft management.

In adult cleft cases pre-surgical orthodontic management include dental decompensation fabrication of appliance that may facilitate certain surgical procedures and post-surgical rehabilitation and settling of occlusion. One of the modes of maxillary management includes antero posterior distraction. With availability of distractor being difficult in rural area, innovation in design of distractor may be of significance. One method is Hyrax expansion screw which is used in trans expansion. A modified design of this proves to be useful in antero posterior distraction.
Corresponding Author:

Dr. Swapnaja Gosavi.

Department of Orthodontics,

Sharad Pawar Dental College,

Sawangi Meghe, Maharashtra,

India.

E-mail: jadhav.swapnaja@gmail.com

DOI: $10.14260 /$ jemds/2020/720

How to Cite This Article:

Gosavi S, Shrivastav S, Kamble R, et al. Multidisciplinary approach for the management of the case of bilateral cleft lip and palate (Baru's score 5) using modified dentoalveolar distractor. J Evolution Med Dent Sci 2020;9(43):3276-3279, DOI: 10.14260/jemds/2020/720

Submission 20-03-2020,

Peer Review 16-09-2020,

Acceptance 23-09-2020,

Published 26-10-2020.

Copyright (C) 2020 Swapnaja Gosavi et al. This is an open access article distributed under Creative Commons Attribution License [Attribution 4.0 International (CC BY 4.0)] 


\section{DISCUSSION OF MANAGEMENT}

\section{Treatment objectives}

- $\quad$ Extraction of over retained 51, 53, 63 and impacted 23.

- Expansion of maxillary arch.

- Initial alignment of maxillary and mandibular arch.

- Correction of cross bite.

- Correction of skeletal class III relationship.

- Improvement of profile.

- To correct macroglossia.

- Prosthetic rehabilitation of missing teeth.

- Post-surgical orthodontic finishing.

Using modified Hyrax as dentoalveolar distractor in antero posterior direction, initially expansion was done by Hyrax screw. The rate of screw opening was once a week. Maxillary and mandibular arches were bonded with MBT 022 slot. The wire sequencing followed was: "0.14 Niti, $0.016 \mathrm{Niti}, 0.016 \times$ 0.22 Niti, $0.016 \times 0.22$ SS, $0.017 \times 0.025$ SS, $0.019 \times 0.025 \mathrm{Niti}$, $0.019 \times 0.025$ stainless Steel". After 8 months post alignment records were taken, and distraction osteogenesis was planned with maxillary arch. Band pinching was done with 16,26,36,46. Custom made Distractor was planned using Hyrax appliance as shown in Figure 3. A month later patient was posted for osteotomy cuts. Distractor was placed intra-operatively. Latency period was 5 days with distraction phase of $1 \mathrm{~mm}$ per day i.e. 2 turns per morning and 2 turns evening. Activation was done for 13 days. Anterior box elastics were given for closure of anterior open bite. After 2 months records were taken, and mandibular setback was planned. Mock surgery was performed and splint was made. Mandibular setback was done by $6 \mathrm{~mm}$ with partial glossectomy.

\section{Treatment Results}

The overall treatment objectives were achieved as shown in Figure 4 and 5. Aesthetic improvement of the frontal and lateral view was evident. At the end of multidisciplinary treatment, the frontal and lateral facial appearances were improved. Maxillary constriction and anterior cross bite were corrected mainly by orthodontic treatment

Correction of "maxillofacial deformities by Orthodontic \& Orthognathic surgical treatment" outcome has been dramatic. The majority of cases show improved aesthetics as well as occlusion, but unless symmetry and balance of relevant muscles, the predictability of long term changes in bone and soft tissue is variable, irrespective of the method.

Distraction osteogenesis is reshaping, lengthening of bones by surgically fracturing it and separation of the bony segments. For the correction of transverse discrepancy expansion is needed. The appliances used expand maxilla in ' $\mathrm{V}$ ' shape with center of rotation around the posterior nasal spine. ${ }^{2}$ Hyrax a RME (Rapid Maxillary Expansion) appliance has the ability to create space as well as it also serves as a retention appliance. In this clinical report we tried to present the use of hyrax as a tool for interdental distraction and that this is not an alternative to Le fort 1 osteotomy. The treatment used in this study was for the correction of skeletal problem. ${ }^{3}$

Maxillary retrognathism, which is generally associated with cleft lip and palate is corrected by Le Fort I osteotomy, has reported that after conventional orthognathic surgery there is a tendency to relapse, where in distraction osteogenesis advances the cleft maxilla, and even after large skeletal movements it has lesser tendency to relapse as "the new bone that is formed in the distraction gap".4

\section{Ways of Doing Maxillary Distraction in Cleft Patients}

1. Through rigid extra oral devices.

2. With the use of internal devices.

3. By surgically assisted maxillary protraction with use of a face mask.

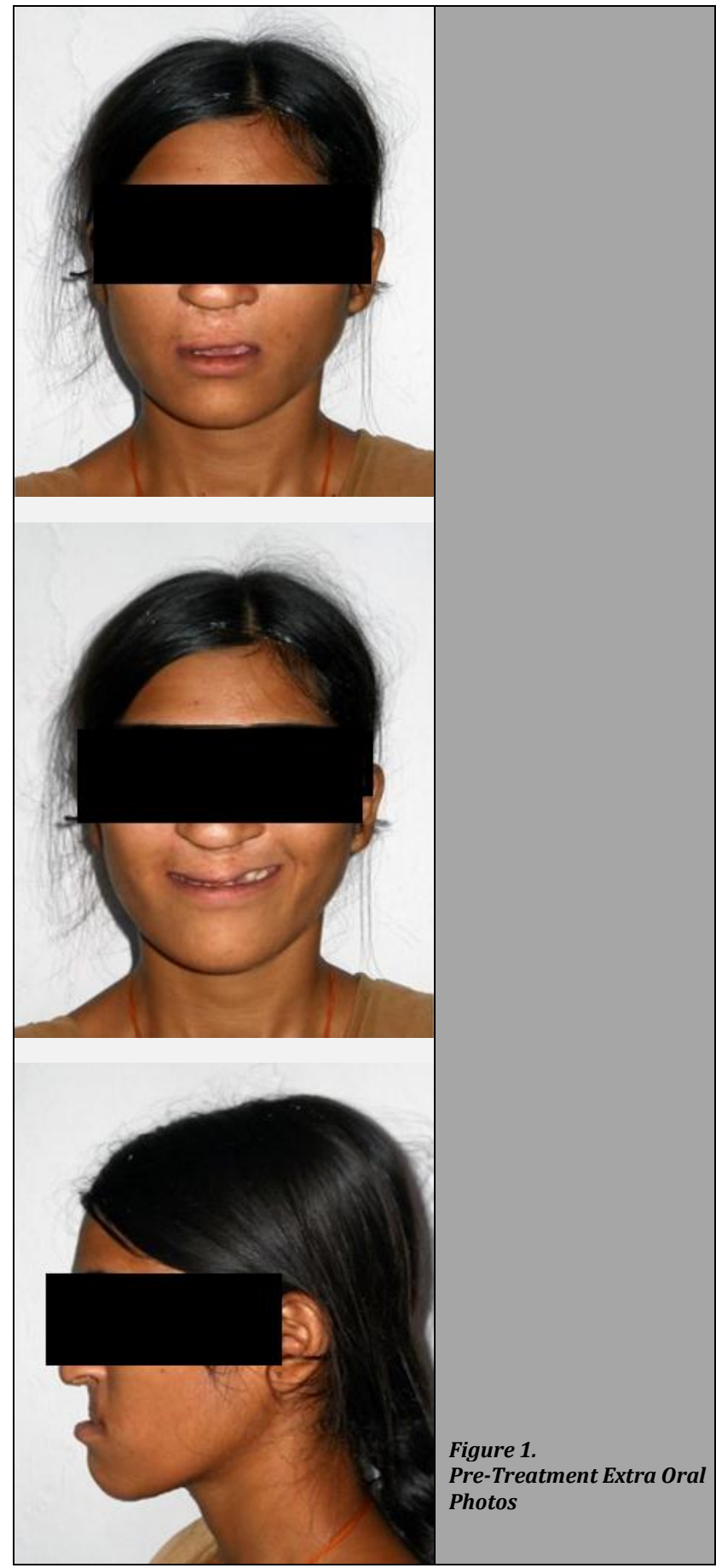

There is an increased stability and growth of maxilla over time when distraction osteogenesis is utilized as a modality in growing patients as was demonstrated by Rachmiel et al. ${ }^{4}$ 
In patients with cleft palate deformities including those with retrusion of the upper jaw, distraction osteogenesis is more advantageous as compared with orthognathic surgeries. This is due to the fact that it facilitates a larger amount of advancement along with a reduced tendency of relapse. In 1957, Prauner and Obwegeser introduced the bilateral sagittal split osteotomy (BSSO) which aided in the correction of transverse, sagittal and vertical positioning of the mandible. ${ }^{5}$ Improvement in the occlusion and facial aesthetics is of prime importance. A higher prevalence of the cleft deformities is seen in the central region of India. ${ }^{6}$

Thus, a multidisciplinary team approach including orthodontists who aid with reverse orthodontics or decompensation prior to surgery as a preoperative procedure is of utmost importance. In approximately $25-60 \%$ of all the patients exhibiting deformities of the cleft lip and palate, surgical intervention is necessary to improve not just facial aesthetics, but also to restore normal functions of the oral cavity. ${ }^{6}$
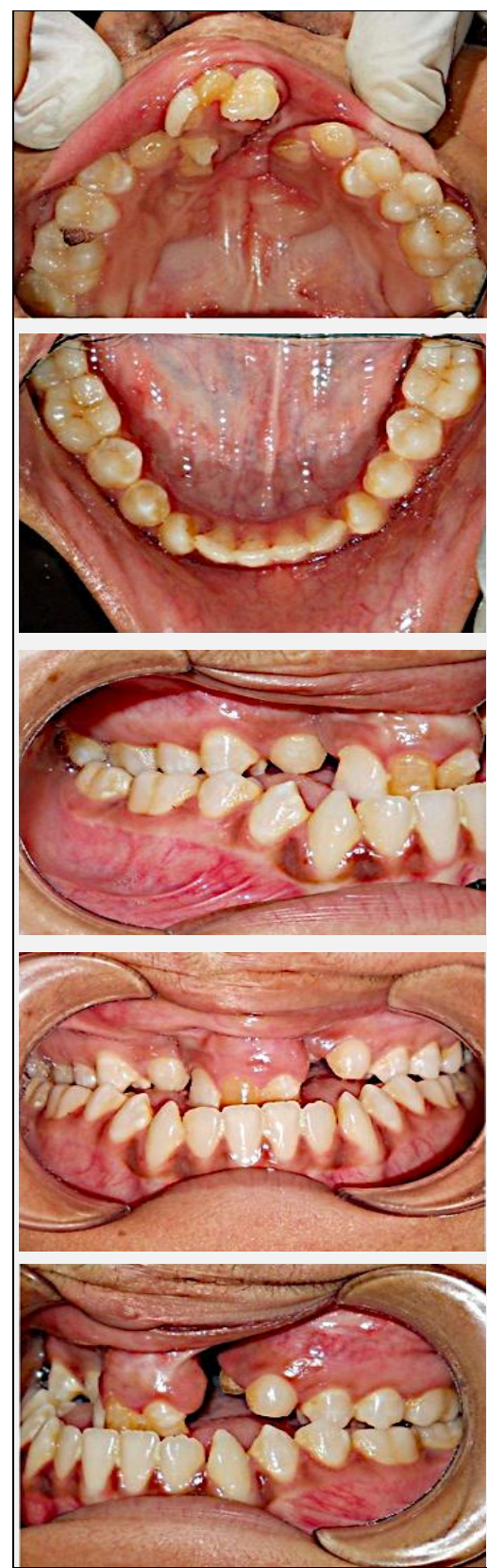

Figure 2.

Pre-Treatment Intra Oral Photos
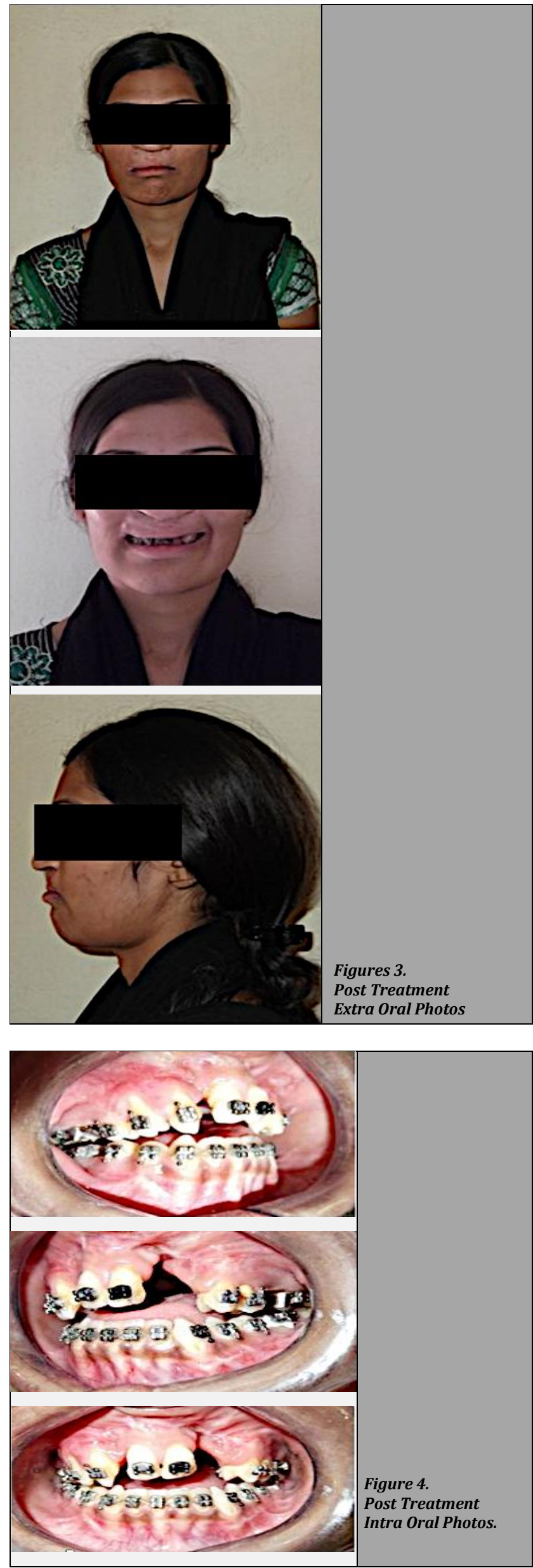


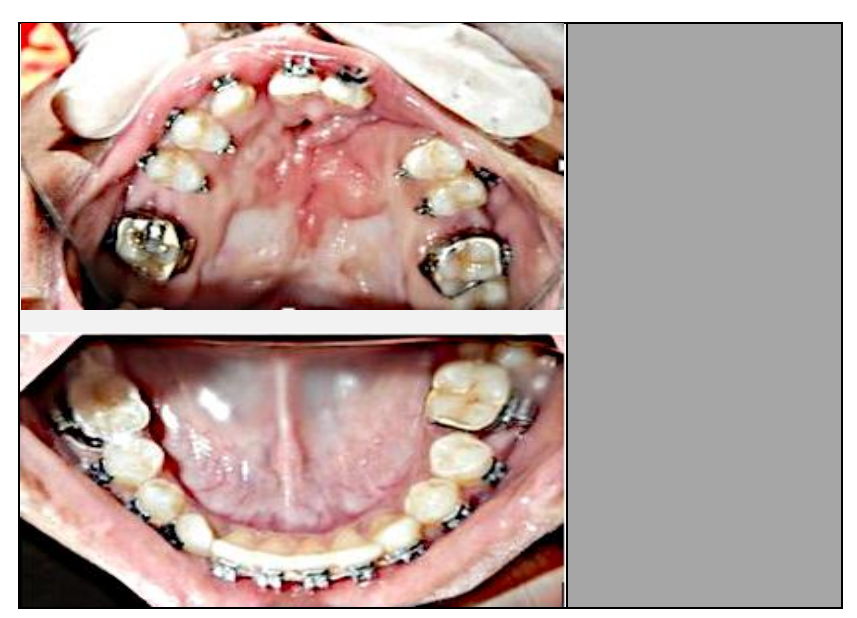

\section{CONCLUSIONS}

Innovative use of easily available instruments may at times be useful as well as economical and will give a desired results with minimum discomfort.

Financial or other competing interests: None.

Disclosure forms provided by the authors are available with the full text of this article at jemds.com.

\section{REFERENCES}

[1] Karia H, Shrivastav S, Karia AK. Three-dimensional evaluation of the airway spaces in patients with and without cleft lip and palate: a digital volume tomographic study. Am J Orthod Dentofacial Orthop 2017;152(3):37181.

[2] Braun S, Bottrel JA, Lee KG, et al. The biomechanics of rapid maxillary sutural expansion. Am J Orthod Dentofacial Orthop 2000;118(3):257-61.

[3] Swennen D, Schliephake H, Dempf R, et al. Craniofacial distraction osteogenesis: a review of the literature: part 1: clinical studies. Int J Oral Maxillofac Surg 2001;30(2):89103.

[4] Lewinson D, Rachmeil A, Rihanai-Bisharat S, et al. Stimulation of Fos- and Jun-related genes during distraction osteogenesis. J Histochem Cytochem 2003;51(9):1161-8.

[5] Trauner R, Obwegeser $H$. The surgical correction of mandibular prognathism and retrognathia with consideration of genioplasty. I. Surgical procedures to correct mandibular prognathism and reshaping of the chin. Oral Surg Oral Med Oral Pathol 1957;10(7):677-89.

[6] Daskalogiannakis J, Mehta M. The need for orthognathic surgery in patients with repaired complete unilateral and complete bilateral cleft lip and palate. Cleft Palate Craniofac J 2009;46(5):498-502. 